\title{
ON BLOW UP OF SOLUTIONS OF NONLINEAR EVOLUTION EQUATIONS
}

\author{
PHILIP KORMAN
}

(Communicated by Kenneth R. Meyer)

\begin{abstract}
We give a complete description of domains of blow up for general second order inequalities, which allows us to obtain some new results on nonexistence of global solutions for nonlinear hyperbolic equations, both in $R^{n}$ and bounded domains.
\end{abstract}

1. Introduction. We study domains of "blow up" of nonlinear evolution equations, i.e., the set of initial data for which the solution goes to infinity in some norm, as time $t$ tends to some finite value. Our main result, Theorem 1, provides (under minimal assumptions) a complete description of the domain of blow up for general second order ordinary differential inequalities. We then use it as a tool to derive some new blow up results for several classes of nonlinear hyperbolic equations, both in $R^{n}$ and in bounded domains.

In [10] H. Levine lists six available techniques for proving blow up of nonlinear evolution equations. All of them involve establishment of a differential inequality for some functional of the solution. One of these techniques "the eigenfunction method" consists in proving blow up of the first Fourier coefficient of solution (the earlier references are S. Kaplan [7], R. Glassey [3]). We illustrate it for the equation $(u=u(x, t))$

$$
u_{t t}-\Delta u=f(u), \quad x \in \Omega, t>0,
$$

with $\Omega$ smooth bounded domain in $R^{n}, n \geq 1, f$ a convex function.

Let $\phi(x)>0$ and $\lambda$ be the principal eigenfunction and eigenvalue respectively of $-\Delta$ on $\Omega$, with $\int_{\Omega} \phi d x=1$. Define $w(t)=\int_{\Omega} u(x, t) \phi(x) d x$. Then using the Jensen's inequality and integration by parts, one easily gets (see [4])

$$
\begin{gathered}
w^{\prime \prime}+\lambda w \geq f(w), \\
w(0)=\int_{\Omega} u(x, 0) \phi(x) d x \equiv w_{1}, \quad w^{\prime}(0)=\int_{\Omega} u_{t}(x, 0) \phi(x) d x \equiv w_{2} .
\end{gathered}
$$

If $\left(w_{1}, w_{2}\right)$ belongs to the domain of blow up of $(* *)$, then no global (i.e., for all $t>0)$ solutions can exist for $(*)$. Theorem 1 below reduces the question to a straightforward analysis of the conservative equation $w^{\prime \prime}+\lambda w=f(w)$.

In $\S 3$ we take up accretive equations of the form $u_{t t}-\Delta u=f\left(u_{t}\right)$, where a similar approach leads to nonconservative differential inequalities. Theorem 2 presents a description of the domain of blow up for $f=u_{t}^{p}, p$ an even integer.

Received by the editors February $18,1987$.

1980 Mathematics Subject Classification (1985 Revision). Primary 35L70.

Key words and phrases. Blow up of solutions, nonlinear evolution equations, phase plane analysis. 
In $\S 5$ we consider a class of nonlinear hyperbolic equations in $R^{n}$, following $\mathrm{T}$. Kato [8]. T. Kato has essentially proved that the conditions (i)-(iii) of $\S 5$ and $p \leq(n+1) /(n-1)$ imply nonexistence of global solutions, provided the initial data satisfies (16). In the Theorem 3 under a stronger restriction $p \leq(n+2) / n$ we show that blow up occurs for a considerably wider set of data, in particular if $\int_{R^{n}} u(x, 0) d x<0$. In Theorem 4 we present a proof of T. Kato's result, partly for completeness, partly since our approach, based on the theory of Emden-Fowler equations (see [2]), appears to be more systematic and simpler.

\section{Blow up of solutions of differential inequalities.}

DEFINITION 1. Consider the differential inequality

$$
w^{\prime \prime} \geq f\left(w, w^{\prime}\right), \quad w(0)=w_{1}, w^{\prime}(0)=w_{2} .
$$

By the domain of blow up for (1) we mean the set of all pairs $\left(w_{1}, w_{2}\right)$, such that any solution of (1) tends to $+\infty$ in finite time. (We consider solutions in the class of piecewise $C^{2}$ functions.)

DEFINITION 2. Let $D$ be some domain in $\left(w, w^{\prime}\right)$ plane. By the shaded region of $D$ we understand $\left\{\left(w, w^{\prime}\right) \mid\left(w, w^{\prime}+\delta\right) \in D\right.$ for some $\left.\delta \geq 0\right\}$. (Think of sun rays coming down parallel to the $w^{\prime}$ axis.) The set of points in the shaded region of $D$, which are not in $D$, will be called the shade of $D$.

THEOREM 1. Assume that the function $f\left(w, w^{\prime}\right)$ is such as to provide local existence, uniqueness and continuous dependence on data for (2) below (e.g., Lipschitz continuous). Let $D$ be the region complementary in $R^{2}$ to the domain of blow up for

$$
w^{\prime \prime}=f\left(w, w^{\prime}\right), \quad w(0)=w_{1}, w^{\prime}(0)=w_{2} .
$$

Then the shaded region of $D$ is complementary in $R^{2}$ to the interior of the domain of blow up for (1). The boundary point will belong to the domain of blow up of (1) iff the trajectory of (2), starting at this point, will stay in the interior of the domain of blow up of (3) for all $t>0$.

ProOF. Part A. We show that some of the solutions of (1), originating in the shaded region of $D$ exist for all time. If the initial point $P$ lies in $D$, there is nothing to prove, since the solution of (2) starting at $P$ is also a solution of inequality (1). So let $P$ lie in the shade of $D$. Notice that the inequality (1) can be written as $\ddot{w}=f(w, \dot{w})+u(t)$, or in the system form as (with $u(t) \geq 0$ )

$$
\dot{w}=v, \quad \dot{v}=f(w, v)+u(t) .
$$

The vector field of $(3), \bar{v}=(v, f(w, v)+u(t))$ has the first component and $f(w, v)$ bounded on compact sets. Also, without loss of generality we may assume $v$ to be of one sign, say negative. Let $R$ be a point in the region $D$ directly over $P$, and $Q$ an arbitrary point in the same component of $D$ to the left of $R$. By choosing $u(t)$ sufficiently large, we can make the vector field $\bar{v}$ point inside the triangle $P Q R$ along the line $P Q$ (and $\bar{v}$ points inside $P Q R$ along $P R$ ). Hence there is a solution of the inequality (1), which starts at $P$ and enters the region $D$ at some time $t=T$. Setting $u(t) \equiv 0$ for $t>T$, we get a global solution for our inequality (1).

Part B. We show that any solution of the inequality (1), originating outside the shaded region of $D$, will blow up in finite time. Rewrite (2) in the system form as

$$
\dot{x}=y, \quad \dot{y}=f(x, y) \text {. }
$$




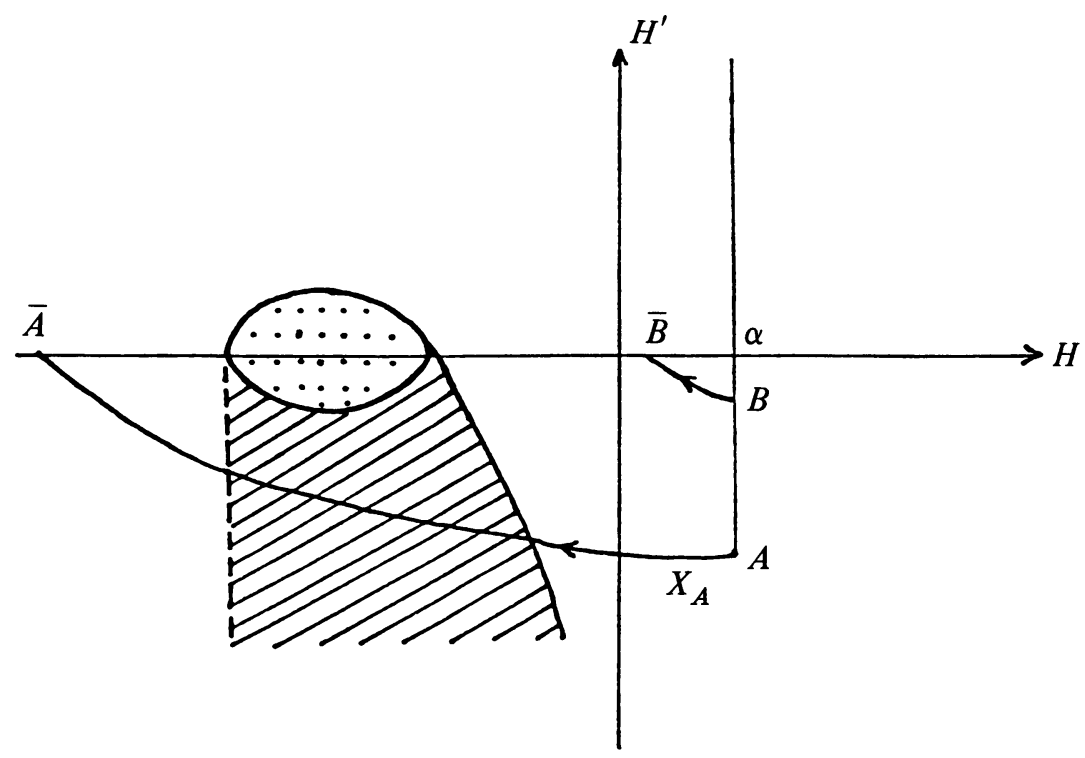

FIGURE 1

By $X_{A}(t)$ and $Y_{A}(t)$ we shall denote the trajectories starting at the point $A$ of (2) and (3), respectively.

Step 1 . We show that any solution of $(2)^{\prime}$, starting outside the shaded region of $D$, will never enter the shade of $D$ (it cannot enter $D$ by definition). Indeed, assume that the trajectory $X_{A}(t)$ goes into the shade of $D$. We may assume that $A$ lies in the lower half plane $y<0$ (otherwise refer to Step 3). Since it blows up, $X_{A}(t)$ must reach the $x$ axis at some point $\bar{A}$, see Figure 1 . Notice that all trajectories of $(2)^{\prime}$, starting on $x=a$ above $A$ blow up, hence they must reach the $x$ axis. If we choose a point $B$ on $x=a$ sufficiently close to the $x$ axis, then by the continuous dependence on data it follows that $X_{B}(t)$ reaches the $x$ axis (at $\bar{B}$ ), while staying in the unshaded region. Now let $A \rightarrow B$, then $\bar{A} \rightarrow \bar{B}$, and the trajectory $X_{A}(t)$ must pass through $D$, which is a contradiction.

Step 2. Since any trajectory $Y_{A}(t)$ lies above $X_{A}(t)$, it follows that $Y_{A}(t)$ will enter the upper half-plane, while staying in the unshaded region.

Step 3. Finally, we show that any solution of (3), starting at a point $A$ in the upper half-plane, outside of shaded region of $D$, blows up in finite time. Similarly to the Step 1 , notice that $X_{A}(t)$ lies above $D$ (which obviously cannot extend to $y=+\infty$, since everything to the left of it, including $A$ would then lie in $D$ ). Hence $Y_{A}(t)$ also lies above $D$. Since along these trajectories both $x(t)$ and $u(t)$ increase monotonically, and $x(t) \rightarrow+\infty$ in finite time, it remains to show that $u(t) \geq x(t)$. For that it suffices to show that if $x\left(t_{1}\right)=u\left(t_{2}\right)$, then $t_{1} \geq t_{2}$. Indeed,

$$
t_{1}=\int_{h_{1}}^{x\left(t_{1}\right)} \frac{d x}{y(x)} \geq \int_{h_{1}}^{u\left(t_{2}\right)} \frac{d u}{v(u)}=t_{2},
$$

which finishes the proof.

EXAMPLE 1. $w^{\prime \prime} \geq w^{p}, w(0)=w_{1}, w^{\prime}(0)=w_{2}(p>1)$. 
The complement to the domain of blow up of $w^{\prime \prime}=w^{p}$ consists of the curve $\Gamma: w^{\prime}=-\sqrt{2} /(p+1) w^{(p+1) / 2}, w \geq 0$. Hence the domain of blow up for $w^{\prime \prime} \geq w^{p}$ is the complement in $R^{2}$ of the shaded region of $\Gamma$ (it includes the ray $w=0, w^{\prime}<0$ ).

3. Blow up for a class of equations in bounded domains. We study the blow up of solutions for the following problem $\left(\Omega \subset R^{n}\right), n \geq 1$,

$$
\begin{aligned}
u_{t t} & =\Delta u+u_{t}^{p}, \quad x \in \Omega, t>0, p \text { an even integer, } \\
u(x, 0) & =g(x), \quad u_{t}(x, 0)=h(x), \\
u(x, t) & =0, \quad x \in \partial \Omega .
\end{aligned}
$$

Let $\phi(x)$ and $\lambda$ denote the principal eigenfunction and eigenvalue of $\Delta \phi+\lambda \phi=0$ in $\Omega, \phi=0$ on $\partial \Omega$, with $\phi>0$ in $\Omega$ and $\int_{\Omega} \phi d x=1$ (which are known to exist under proper assumptions on $\Omega$ ). Letting as before $w=\int_{\Omega} u \phi d x$, integrating by parts and using the Hölder's inequality, we get

$$
\begin{aligned}
w^{\prime \prime} & \geq-\lambda w+{w^{\prime}}^{p} \\
w(0) & \equiv \int_{\Omega} g(x) \phi(x) d x \equiv w_{1}, \quad w^{\prime}(0)=\int_{\Omega} h(x) \phi(x) d x \equiv w_{2},
\end{aligned}
$$

and we are interested in the domain of blow up for (5).

The case $p=2$ is special, since the equation $w^{\prime \prime}=-\lambda w+w^{\prime^{2}}$ can be integrated explicitly. First, by stretching time (this does not affect the blow up of solutions) we reduce it to

$$
w^{\prime \prime}=-w+w^{\prime^{2}} .
$$

Letting $w^{\prime}=p(w), w^{\prime \prime}=p^{\prime} p$, we get $\left(p^{2} / 2\right)^{\prime}=-w+p^{2}$, which is a linear equation for $p^{2}$. Integrating, we get the phase curves for (6)

$$
w^{\prime}= \pm \sqrt{c e^{2 w}+w+\frac{1}{2}}
$$

The constant $c$ is determined by the initial data, $c=\left(w_{2}^{2}-w_{1}-\frac{1}{2}\right) e^{-2 w_{1}}$. (Notice, $c \geq-\frac{1}{2}$ for any $w_{1}, w_{2}$.) It is easy to see that solutions of (6) behave as follows.

(i) $c>0$, blow up in finite time.

(ii) $c=0$, solution goes to infinity in infinite time.

(iii) $-\frac{1}{2}<c<0$, solutions are periodic.

(iv) $c=-\frac{1}{2}$, equilibrium point at the origin.

One sees that the curve $\Gamma: w^{\prime}= \pm \sqrt{w+\frac{1}{2}}$ separates the regions of blow up and global existence. Hence the blow up region for (5) with $p=2$ is the complement in $R^{2}$ of $\left\{\left(w_{1}, w_{2}\right) \mid w_{2} \leq \sqrt{w_{1}+\frac{1}{2}},-\frac{1}{2}<w_{1}<\infty\right\} \cup\left(-\frac{1}{2}, 0\right)$.

We show next that the situation is similar for $p=2 k, k \geq 1$. By stretching of independent and dependent variables, we may write (5) as $\ddot{x}=-x+\dot{x}^{2 k}$, or in system form

$$
\dot{x}=y, \quad \dot{y}=-x+y^{2 k}, \quad k>1 .
$$

THEOREM 2. There exists a trajectory $\Gamma$ of (8), separating the blow up and global existence regions. It is symmetric with respect to the $x$-axis, intersecting it once at $x_{0}<0$. Along $\Gamma$ both $x$ and $y$ tend to $\infty$ as $t \rightarrow \pm \infty$.

PROOF. We begin by noticing symmetry: if $(x(t), y(t))$ is an integral curve of $(8)$, so is $(x(-t),-y(-t))$. Hence the phase portrait of $(8)$ is symmetric with 
respect to the $x$-axis. Consider the vector field of (8), $\bar{v}=\left(y,-x+y^{2 k}\right)$ on the curves $\Gamma_{1}:-x+y^{2 k}=1$, and $\Gamma_{2}:-x+y^{2 k}=0$ in the first quadrant. It is easy to see that $\bar{v}$ points above (the tangent of) $\Gamma_{1}$ and below of $\Gamma_{2}$. This means that there exist solutions of (8) in $D$ (see e.g. [5]). Call $\Gamma$ the highest of the trajectories trapped in the region $D$ between $\Gamma_{1}$ and $\Gamma_{2}$.

Notice that its $y$ intercept in the first quadrant $y_{0} \neq 0$ ( $\Gamma$ cannot enter the origin, since by $\left.(8), \lim _{x \rightarrow 0+} d y / d x \neq+\infty\right)$. When traced back in time, $\Gamma$ will decrease monotonically in $x$ and $y$, and intersect the $x$-axis at some $x_{0}<0$. The symmetry with respect to the $x$-axis gives us the rest of $\Gamma$ by reflection.

Since in the region $D, \dot{x} \leq(x+1)^{1 / 2 k}$, it follows that $\Gamma$ is a global solution, and hence so are all trajectories to the right of $\Gamma$ (they travel slower than $\Gamma$ ). It remains to show that all trajectories to the left of $\Gamma$ blow up. Indeed, after a finite time any such trajectory will enter the region $D_{1}: y>(x+1)^{1 / 2 k}, x>0$ (above $\Gamma_{1}$ ). Any point in $D_{1}$ will lie to the left of the curve $\Gamma_{\varepsilon}: x=(1-\varepsilon) y^{2 k}$ if $\varepsilon$ is sufficiently small, i.e., it will lie in the region $D_{\varepsilon} \equiv D_{1} \cap\left(x<(1-\varepsilon) y^{2 k}\right)$. We show next that the trajectories never leave $D_{\varepsilon}$. The curves $\Gamma_{1}$ and $\Gamma_{\varepsilon}$ intersect at $y=\varepsilon^{-1 / 2 k}$. On $\Gamma_{\varepsilon}$ the vector field is $\bar{v}=\left(y, \varepsilon y^{2 k}\right)$ with the slope $\varepsilon y^{2 k-1}$. The slope of tangent on $\Gamma_{\varepsilon}$ is $1 / 2 k(1-\varepsilon) y^{2 k-1}$. And it is easy to see that $\varepsilon y^{2 k-1}>1 / 2 k(1-\varepsilon) y^{2 k-1}$ for $y>\varepsilon^{-1 / 2 k}$, provided $\varepsilon$ is sufficiently small. This implies that trajectories cannot leave through $\Gamma_{\varepsilon}$, and since they cannot leave through $\Gamma_{1}$ too, they must remain in $D_{\varepsilon}$.

In $D_{\varepsilon}$ we have

$$
\dot{y}=-x+y^{2 k} \geq-(1-\varepsilon) y^{2 k}+y^{2 k}=\varepsilon y^{2 k},
$$

and hence no global solutions are possible. We see that $\Gamma$ separates the blow up and stability regions, and the proof is complete.

4. Nonexistence of global solutions for a class of hyperbolic equations in $R^{n}$. We consider nonlinear hyperbolic equations

$$
\begin{aligned}
u_{t t}-A u & =f(x, t, u), \quad x \in R^{n}, t>0, \\
u(x, 0) & =g(x), \quad u_{t}(x, 0)=h(x) .
\end{aligned}
$$

Here $A$ is a uniformly elliptic operator in divergence form,

$$
A=\sum_{j, k=1}^{n} \frac{\partial}{\partial x_{j}} a_{j k}(x, t) \frac{\partial}{\partial x_{k}}-\sum_{j=1}^{n} \frac{\partial}{\partial x_{j}} a_{j}(x, t) .
$$

Throughout this section we assume the following.

(i) The data $g(x), h(x)$ are of compact support, supported say in $|x|<1$. We also assume that for $f \equiv 0$ equation (9) has propagation speed not greater than one, i.e., $u(x, t)$ is supported in $|x|<t+1$.

(ii) $f(x, t, 0) \equiv 0$. This guarantees us that at time $t$ solution of (9) is supported in $|x|<t+1$.

(iii) $f(x, t, u) \geq c_{0}|u|^{p}, p>1, c_{0}=$ const $>0$.

We do not make any explicit smoothness assumptions on $a_{j k}, a_{j}, f, g, h$, since we are not interested in the existence problem here. Instead, we make assumptions on the solution $u$, such as the finite propagation speed and regularity, $u \in C^{2}\left(R^{n} \times(0, \infty)\right)$. Define $w(t)=\int_{R^{n}} u(x, t) d x$. Notice that $w(0)=\int_{R^{n}} g(x) d x, w^{\prime}(0)=\int_{R^{n}} h(x) d x$. 
Integrating equation (9) over $R^{n}$, using (iii) and that $u$ is of compact support, we get

$$
w^{\prime \prime} \geq c_{0} \int_{R^{n}}|u|^{p} d x .
$$

Since $u(x, t)$ is supported in the ball $|x|<t+1$ of volume $c_{n}(t+1)^{n}$, we estimate $(1 / p+1 / q=1)$

$$
|w|=\left|\int_{R^{n}} u(x, t) d x\right| \leq\left(\int_{R^{n}}|u|^{p} d x\right)^{1 / p} c_{n}^{1 / q}(t+1)^{n / q},
$$

which combined with (10) gives

$$
w^{\prime \prime} \geq c_{1}(t+1)^{-n p / q}|w|^{p} \quad\left(c_{1}=c_{0} / c_{n}^{p / q}\right) .
$$

Assuming now $p \leq(n+1) /(n-1)$ for $n>1$, and automatically for $n=1$, we get

$$
w^{\prime \prime} \geq c_{1}(t+1)^{-p-1}|w|^{p} \text {. }
$$

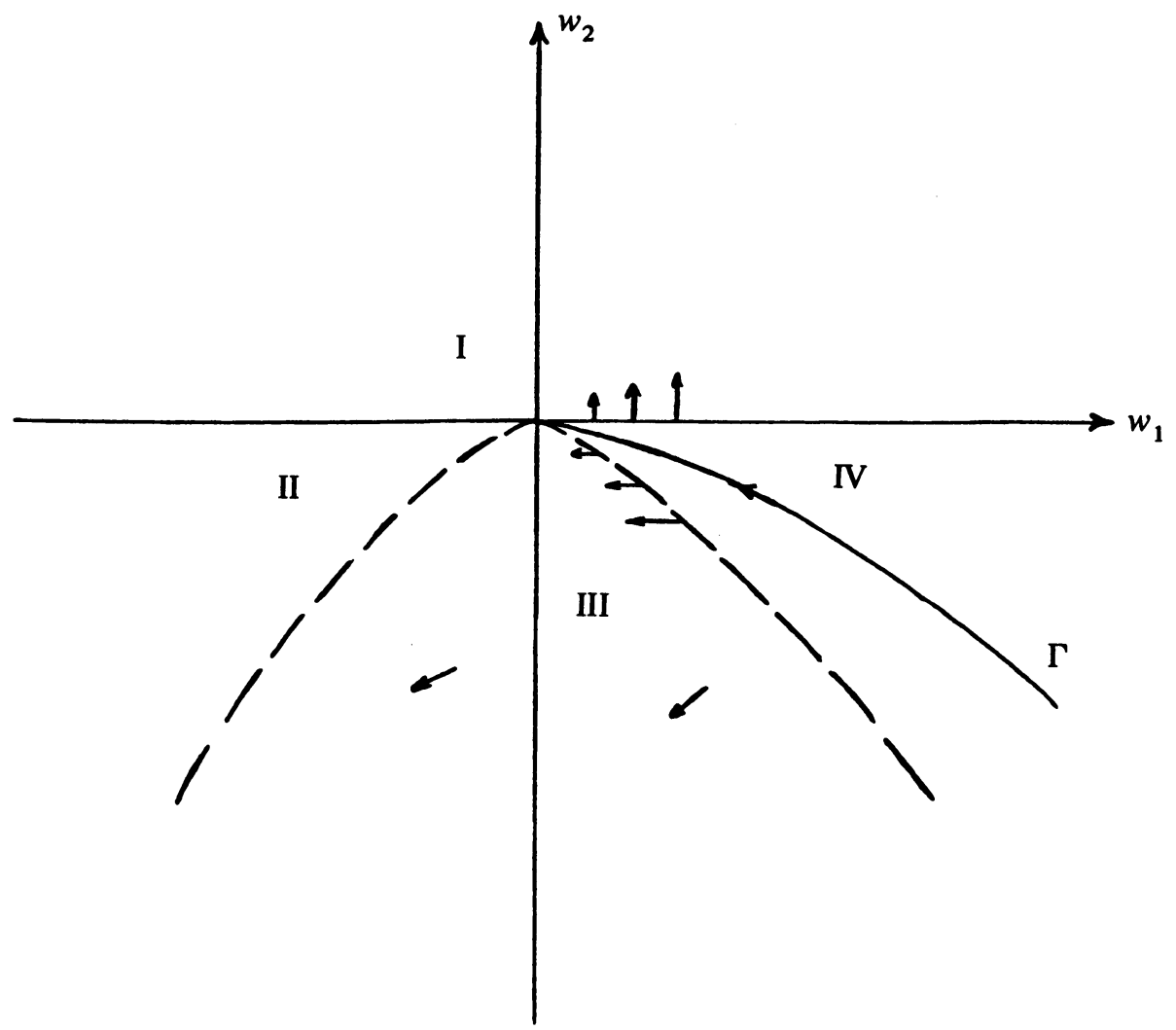

FIGURE 2

THEOREM 3. For the problem (9) assume (i), (ii), (iii) and $1<p \leq(n+2) / n$. Denote $w_{1}=\int_{R^{n}} g(x) d x, w_{2}=\int_{R^{n}} h(x) d x$. Then there exists a trajectory $\Gamma$ of the equation $w^{\prime \prime}-w^{\prime}=c_{1} w^{p}\left(c_{1}\right.$ as in (12)), joining the origin with $(\infty,-\infty)$ in 
the $\left(w, w^{\prime}\right)$ phase plane, such that if $\left(w_{1}, w_{2}\right)$ lies outside of the shaded region of $\Gamma$, then (9) cannot have a global classical solution.

PROOF. Since $-n p / q \geq-2$ for $p \leq(n+2) / n$, it follows from (12) that

$$
w^{\prime \prime} \geq c_{1}(t+1)^{-2}|w|^{p}, \quad w(0)=w_{1}, w^{\prime}(0)=w_{2} .
$$

Let now $\tau=\log (t+1)$. Then (13) becomes

$$
w_{\tau \tau}-w_{\tau} \geq c_{1}|w|^{p}, \quad w(0)=w_{1}, w_{\tau}(0)=w_{2} .
$$

We proceed to show that the domain of blow up for (14) is as described in the theorem. First, we describe the domain of blow up for $w_{\tau \tau}-w_{\tau}=c_{1}|w|^{p}$, or in system form

$$
\begin{aligned}
w_{\tau} & =v, & w(0) & =w_{1}, \\
v_{\tau} & =c_{1}|w|^{p}+v, & v(0) & =w_{2} .
\end{aligned}
$$

We divide the $(w, v)$ phase plane into four regions; see Figure 2.

(i) Domain I, $\left\{\left(w_{1}, w_{2}\right), w_{2}>0\right.$ or $\left.w_{2}=0, w_{1} \neq 0\right\}$. In this case $w_{\tau \tau} \geq c_{1}|w|^{p}$, and hence all trajectories starting in I, blow up in finite time by Example 1.

(ii) Domain II, $\left\{\left(w_{1}, w_{2}\right), w_{1}<0,-c_{1}\left|w_{1}\right|^{p} \leq w_{2}<0\right\}$. Trajectories starting here move northwest, and enter Domain I in finite time.

(iii) Domain III, $\left\{\left(w_{1}, w_{2}\right),-\infty<w_{1}<\infty, w_{2}<-c_{1}\left|w_{1}\right|^{p}\right\}$. In this domain trajectories move southwest with the slope $c_{1}|w|^{p}+v / v \leq 1$. Hence, they must enter Domain II in finite time.

(iv) Domain IV, $\left\{\left(w_{1}, w_{2}\right), w_{1}>0,-c_{1} w_{1}^{p} \leq w_{2}<0\right\}$. Trajectories leave Domain IV through every point of its boundary. Hence there exists a trajectory $\Gamma$, entering the origin. It is unique, since nonuniqueness would imply that the vector field (whose divergence is one) contracts some volumes, which is impossible by the Liouville's theorem. Trajectories above $\Gamma$ enter Domain I, and the ones below it enter Domain IV in finite time. Hence $\Gamma$ is the complement of the domain of blow up for (15), and so the proof is complete in view of the Theorem 1 (the ray $w_{1}=0$, $w_{2}<0$ belongs to the domain of the blow up).

THEOREM 4 (Essentially of T. Kato [8]). Assume $1 \leq p \leq(n+1) /(n-1)$, and

$$
\text { either } w^{\prime}(0)>0 \text {, or } w^{\prime}(0)=0 \text { and } w(0) \neq 0 \text {. }
$$

Then inequality (12) has no global solutions. (And hence (9) has no global classical solution.)

PrOOF. First notice that $w^{\prime}$ is a nondecreasing function; then $w^{\prime}(t)>0$ for $t>0$, since by (16) either $w^{\prime}(0)>0$, or $w^{\prime}(0)=0$ and $w^{\prime \prime}(0)>0$. Hence, as $t \rightarrow+\infty$ we have only two possibilities: (a) $w^{\prime} \rightarrow a=$ const $>0$, (b) $w^{\prime} \rightarrow+\infty$.

In case (a) we have $w \sim$ at as $t \rightarrow+\infty$, but then

$$
w^{\prime \prime} \geq c_{1}(t+1)^{-p-1}\left(\frac{1}{2} \text { at }\right)^{p} \geq c_{2}(1 / t) \text { for large } t,
$$

i.e., $w \geq c_{3} t \log t$ for large $t$, a contradiction. 

(12)

In case (b), $w \geq A t$ for any $A>0$, eventually (i.e., if $t$ is large enough). From

$$
\begin{aligned}
w^{\prime} w^{\prime \prime} & \geq c_{1} / p+1\left[(t+1)^{-p-1} w^{p+1}\right]^{\prime}+c_{1}(t+1)^{-p-2} w^{p+1} \\
& \geq c_{4}\left[(t+1)^{-p-1} w^{p+1}\right]^{\prime} .
\end{aligned}
$$

Integrating, we get

$$
w^{2} \geq 2 c_{4} t^{-p-1} w^{p+1}-c_{5}=2 c_{4}\left(\frac{w}{t}\right)^{p-1}\left(\frac{w}{t}\right)^{2}-c_{5} \geq A_{1}\left(\frac{w}{t}\right)^{2}
$$

for arbitrary large $A_{1}$, eventually. Integrating (17), we see that $w \geq t^{k}$ for arbitrary large $k$, eventually. Returning to (17), we see that $w^{\prime} \geq c_{6} w^{1+(p-1) / 4}$, eventually. The last inequality obviously cannot have global solutions with $w>0$, which completes the proof.

REMARK. Our approach makes the proof of blow up especially simple if $p<$ $(n+1) /(n-1)$. Indeed, in this case we consider instead of (12) inequality (11) with $-n p / q>-p-1$. Then, starting in case (b) with $w \geq A t$ ( $A$ arbitrary large), by iterating (11) we see that eventually $w \geq t^{k}$ for any $k>0$, but then from (12), $w^{\prime \prime} \geq|w|^{(p+1) / 2}$ and the blow up is obvious (see Example 1).

Limitations of the method. The proof of blow up depended in a crucial way on finite propagation speed, which is a major limitation of the method. For example, for operators of the form

$$
\left(\frac{\partial}{\partial t}\right)^{m} u+\sum_{j<m} a_{\nu, j}\left(\frac{\partial}{\partial x}\right)^{\nu}\left(\frac{\partial}{\partial t}\right)^{j} u=0
$$

it would require that $|\nu|+j \leq m$, see [11].

Finally, we show that T. Kato's power $p=(n+1) /(n-1)(n>1)$ is the best obtainable by this technique, namely for $p>(n+1) /(n-1)$ inequality (11) may have global solutions with $w^{\prime}(0)>0$. Indeed, if $p=(n+1) /(n-1)+\varepsilon, \varepsilon>0$, then it is easy to check that (11) (with equal sign) has a solution $w(t)=\beta(t+1)^{\alpha}$, where

$$
\alpha=\frac{2+\varepsilon n(n-1)}{2+\varepsilon(n-1)}>1 \text { and } \beta=\left(\frac{\alpha(\alpha-1)}{c_{1}}\right)^{1 /(p-1)}
$$

However, the power $p=(n+1) /(n-1)$ is not optimal (e.g., for $n=3$ solutions blow up, provided $1<p<\sqrt{2}+1$, as follows from F. John [6]).

ACKNOWLEDGMENTS. I wish to thank M. Levi for his very substantial help on Theorem 1, and J. Greenberg for his interest in my work.

\section{REFERENCES}

1. J. M. Ball, Remarks on blow-up and nonexistence theorems for nonlinear evolution equations, Quart. J. Math. Oxford (2) 28 (1977), 473-486.

2. R. Bellman, Stability theory of differential equations, McGraw-Hill, New York, 1953.

3. R. Glassey, Blow-up theorems for nonlinear wave equations, Math. Z. 132 (1973), 183-203.

4. __ Finite-time blow-up for solutions of nonlinear wave equations, Math. Z. 177 (1981), 323340.

5. J. K. Hale, Ordinary differential equations, Wiley, New York, 1969.

6. F. John, Blow-up of solutions of nonlinear wave equations in three space dimensions, Manuscripta Math. 28 (1979), 235-268. 
7. S. Kaplan, On growth of solutions of quasilinear parabolic equations, Comm. Pure Appl. Math. 16 (1957), 305-330.

8. T. Kato, Blow up of solutions of some nonlinear hyperbolic equations, Comm. Pure Appl. Math. 33 (1980), 501-505.

9. S. Klainerman and G. Ponce, Global, small amplitude solutions to nonlinear evolution equations, Comm. Pure Appl. Math. 36 (1983), 133-141.

10. H. A. Levine, Nonexistence of global weak solutions to some properly and improperly posed problems of mathematical physics: the method of unbounded Fourier coefficients, Math. Ann. 214 (1975), 205-220.

11. S. Mizohata, The theory of partial differential equations, Cambridge Univ. Press, London and New York, 1973.

Department of Mathematical Sciences, University of Cincinnati, CincinNATI, OHIO 45221-0025 